\title{
Exosomes as a biomarker platform for detecting epidermal growth factor receptor-positive high-grade gliomas
}

\author{
Sasidhar Venkata Manda, MSc, PhD, ${ }^{1}$ Yogesh Kataria, MBBS, ${ }^{2}$ Babul Reddy Tatireddy, MSc, ${ }^{1}$ \\ Balasubramaniam Ramakrishnan, MSc, ${ }^{1}$ Boola Gnana Ratnam, MBBS, MCh, ${ }^{2}$ \\ Rahul Lath, MBBS, MCh, DNB, ${ }^{2}$ Alok Ranjan, MBBS, MCh, ${ }^{2}$ and Amitava Ray, MBBS, MD, FRCS 1,2 \\ ${ }^{1}$ Apollo Hospitals Educational and Research Foundation (AHERF); and 2Department of Neurosurgery, Apollo Hospitals, \\ Hyderabad, Telangana, India
}

OBJECTIVE High-grade glial brain tumors are often characterized by an elevated expression of the tumorigenic epidermal growth factor receptor variant III (EGFRvIII). The authors sought to establish a clinically adaptive protocol as a noninvasive diagnostic tool for EGFRvIll detection through serum exosomes.

METHODS Purity of serum exosome/RNA was confirmed by electron microscopy and flow cytometry and through an RNA bioanalyzer profile. EGFRvIII amplification was initially established by semiquantitative polymerase chain reaction in tumor tissues and exosomes. Diagnostic performance of EGFRvIII transcript in tissue versus exosome was determined using a $2 \times 2$ clinical table approach. Overall survival was determined using Kaplan-Meier analysis.

RESULTS The EGFRvIll transcript was detected in $39.5 \%$ of tumor tissue samples and in $44.7 \%$ of their paired serum exosome samples; $28.1 \%$ of biopsy tumors coexpressed wild-type EGFR and EGFRvIII. Tissue EGFRvIII amplification served as the reference-positive control for its paired serum expression. The overall clinical sensitivity and specificity of semiquantitative exosome EGFRvIll polymerase chain reaction detection assay in serum were $81.58 \%(95 \% \mathrm{Cl} 65.67 \%$ $92.26 \%)$ and $79.31 \%(95 \% \mathrm{Cl} 66.65 \%-88.83 \%)$, respectively. Age, sex, tumor location, and side of the body on which the tumor was located had no effect on the detection rate of exosomal EGFRvIII transcript. EGFRvIII expression either in exosomes or tissue correlated with poor survival.

CONCLUSIONS The authors established a serum-based method for detection of EGFRvIII in high-grade brain tumors that might serve as an optimal noninvasive method for diagnosing EGFRvIII-positive high-grade gliomas.

https://thejns.org/doi/abs/10.3171/2016.11.JNS161187

KEY WORDS exosomes; EGFRVIII; liquid biopsy; circulatory biomarkers; oncology

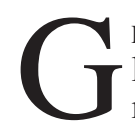
LIOMAS account for approximately $80 \%$ of all malignant brain tumors, of which $54 \%$ are glioblastomas (GBMs). ${ }^{15}$ Primary GBMs develop rapidly and have a very short clinical history with a median survival of approximately 1 year, even after the standard of care treatment. ${ }^{9,27}$ Pathologically, GBMs are the most invasive and aggressive of the brain tumors, with marked nuclear atypia, mitosis, neovascular proliferation, and necrosis, often occurring in focal areas and contributing to tumor heterogeneity and difficulties in pathological diagnosis. ${ }^{15}$

Although neuroimaging may be an important modality in the diagnosis of high-grade tumors, its usage is compromised, as GBMs share common radiological features with other primary tumors, abscesses, and metastases. Moreover, despite the recent advances in surgical care, there is a small but significant $(5 \%-10 \%)$ morbidity associated with

ABBREVIATIONS EGFR = epidermal growth factor receptor; EGFRVIII = epidermal growth factor receptor variant III; GAPDH = glyceraldehyde 3-phosphate dehydrogenase; $\mathrm{GBM}=$ glioblastoma; NPV = negative predictive value; $\mathrm{OS}$ = overall survival; $\mathrm{PCR}=$ polymerase chain reaction; $\mathrm{PPV}=$ positive predictive value; $\mathrm{RT}=$ reverse transcription; TCGA = The Cancer Genome Atlas; TEM = transmission electron microscopy.

SUBMITTED May 14, 2016. ACCEPTED November 7, 2016.

INCLUDE WHEN CITING Published online June 2, 2017; DOI: 10.3171/2016.11.JNS161187. 
invasive tumor biopsy. ${ }^{36}$ Therefore, there is a critical need for the development of blood-based biomarkers that can be used not only in diagnosis, but also for monitoring disease progression after surgery.

The Cancer Genome Atlas (TCGA) has divided GBMs into 4 distinct subgroups: proneural, classical, neural, and mesenchymal, based on genetic, epigenetic, and transcriptional variations (https://cancergenome.nih.gov). ${ }^{28}$ Classical GBM is the most common TCGA subgroup and is characterized by the presence of elevated levels of expression of epidermal growth factor receptor $(E G F R)$ and its variants. ${ }^{35}$ In approximately $50 \%-60 \%$ of these classical tumors, point mutations and/or EGFRvIII (a result of the deletion of exons 2-7 of wild-type $E G F R$ ) are prevalent. ${ }^{33}$ EGFRvIII is exclusively present in patients with malignant tumors and not in healthy individuals, ${ }^{5}$ making it an attractive target for diagnosis. EGFRvIII signaling has been proven to promote tumorigenesis and angiogenesis, and even confer resistance to radiation therapy and chemotherapy ${ }^{24} \mathrm{~A}$ tumor-specific origin of EGFRvIII is highlighted by its downregulated expression in patients after surgical tumor removal. ${ }^{32}$ Thus, detection of EGFRvIII is indicative of a high-grade glioma and can define GBM diagnosis even in the absence of histological criteria, ${ }^{12}$ and hence it can play an important role in clinical diagnosis. ${ }^{3}$

Exosomes are nanovesicles ranging from 30 to $100 \mathrm{~nm}$ in diameter that are formed by plasma membrane invagination of late endosomes. ${ }^{6}$ Exosomes are present in all major body fluids. ${ }^{17}$ It has been shown that exosomes play important roles not only in in cell signaling and signal transduction but also in transporting intracellular cargo, including protein, DNA, RNA, and other metabolites. ${ }^{34}$ Exosomal components represent the tumor cellular transcriptome and can be used for molecular profiling in real time. ${ }^{23}$ The use of exosomal cargo as a source of biomarkers may circumvent limitations and challenges associated with current histological diagnosis of gliomas. ${ }^{21}$ Recent studies have demonstrated the presence of EGFRvIII transcripts in cell culture supernatants of primary GBM cultures and plasma components of GBM patients, ${ }^{32}$ as well as platelet RNA, ${ }^{26}$ that host GBM oncosomal RNA.

Presently, clinical validation of EGFRvIII detection through liquid biopsy methods is lacking. In this study we sought to establish a serum-based method of detecting exosome EGFRvIII that might help in the diagnosis of high-grade gliomas, as per recommended guidelines for tumor marker prognostic studies (REMARK), ${ }^{2}$ taking into consideration the possible impact of sex, age, and anatomical location of the tumors.

\section{Methods}

\section{Sample Collection and Assay Establishment}

The Apollo Hospitals ethics committee granted approval for this prospective and blinded study. Written informed consent was obtained from all study participants. Tumor biopsies and paired serum samples from 96 adult patients with histologically confirmed high-grade glioma who had undergone craniotomy were obtained from Apollo Hospital, Hyderabad. Sera obtained from patients with other neurological diseases (meningioma, tuberculous abscess, brain injury, and stroke) were used as controls. High-grade breast cancer samples were used as a positive source for detection of EGFRvIII. ${ }^{8}$ U87MG (a high-grade glioblastoma cell line; a kind gift from Dr. E. SreeKumar, Rajiv Gandhi Centre for Biotechnology, Kerala, India) was used as a positive control for wild-type EGFR. This cell line was procured initially from ATCC (lot no. 59681610; purchased on October 19, 2012); no additional authentication was performed on the cell line after obtaining it from ATCC. EGFRvIII 4.12 clone cell line, a positive cell line for EGFRvIII, was procured from Celther, Polska, and was used as a positive control for EGFRvIII assay establishment. No additional authentication was performed on the cell line after obtaining it from the company.

Blood samples were collected from 50 healthy volunteers in the Apollo Hospital community and served as negative controls to detect EGFRvIII expression. Biopsy tissue was flash frozen/collected in RNAlater (ThermoFisher Scientific). All tissue sample aliquots were stored in liquid nitrogen. All serum samples were stored as multiple aliquots at $-80^{\circ} \mathrm{C}$.

\section{Study Design}

Samples obtained from 96 high-grade glioma patients, 15 patients with other neurological diseases, and 50 age- and sex-matched healthy volunteers were used in this blinded study (Fig. 1). Tumor tissue and paired serum samples were collected from the operating room directly at the time of surgery. Tumor tissue was stored in RNAlater, and serum was stored at $-80^{\circ} \mathrm{C}$. After an initial optimization of the assay using U87 and U87-EGFRvIII cell lines for wild-type EGFR and EGFRvIII, the PCR assay was run on the 96 tumor and exosome RNA samples along with the healthy and neurological controls. All experiments were repeated at least 3 times.

The overall study design is shown in Fig. 1. Statistical analysis for estimating the diagnostic performance of the exosome EGFRVIII PCR assay was performed. Overall survival (OS) was calculated. The effects of covariates (e.g., age, sex, and anatomical tumor location and side) were also analyzed.

\section{Exosomal Handling and Storage}

Paired serum samples were stored as multiple aliquots at $-80^{\circ} \mathrm{C}$. Serum samples were thawed at $37^{\circ} \mathrm{C}$ in a water bath and centrifuged in 3 steps, $600 \mathrm{~g} / 10 \mathrm{~min}, 2000 \mathrm{~g} / 20$ $\mathrm{min}$, and finally at $10,000 \mathrm{~g} / 20 \mathrm{~min}$, to acquire a cell $/ \mathrm{pel}-$ let-free clarified serum. Exosomes were precipitated from the clarified serum using the Total Exosome Isolation Kit (ThermoFisher Scientific) according to the manufacturer's instructions. The typical serum volume used for RNA isolation was around 2-3 ml per sample. Serum samples were incubated with the precipitating reagent at $4^{\circ} \mathrm{C}$ for 30 minutes and then centrifuged at room temperature at $10,000 \mathrm{~g}$ for 10 minutes. Supernatant was discarded, and the exosome pellet was suspended in the exosome resuspension buffer and immediately used for assays. Protein quantification was performed using a conventional bicinchoninic acid assay (BCA) kit (ThermoFisher Scientific). 


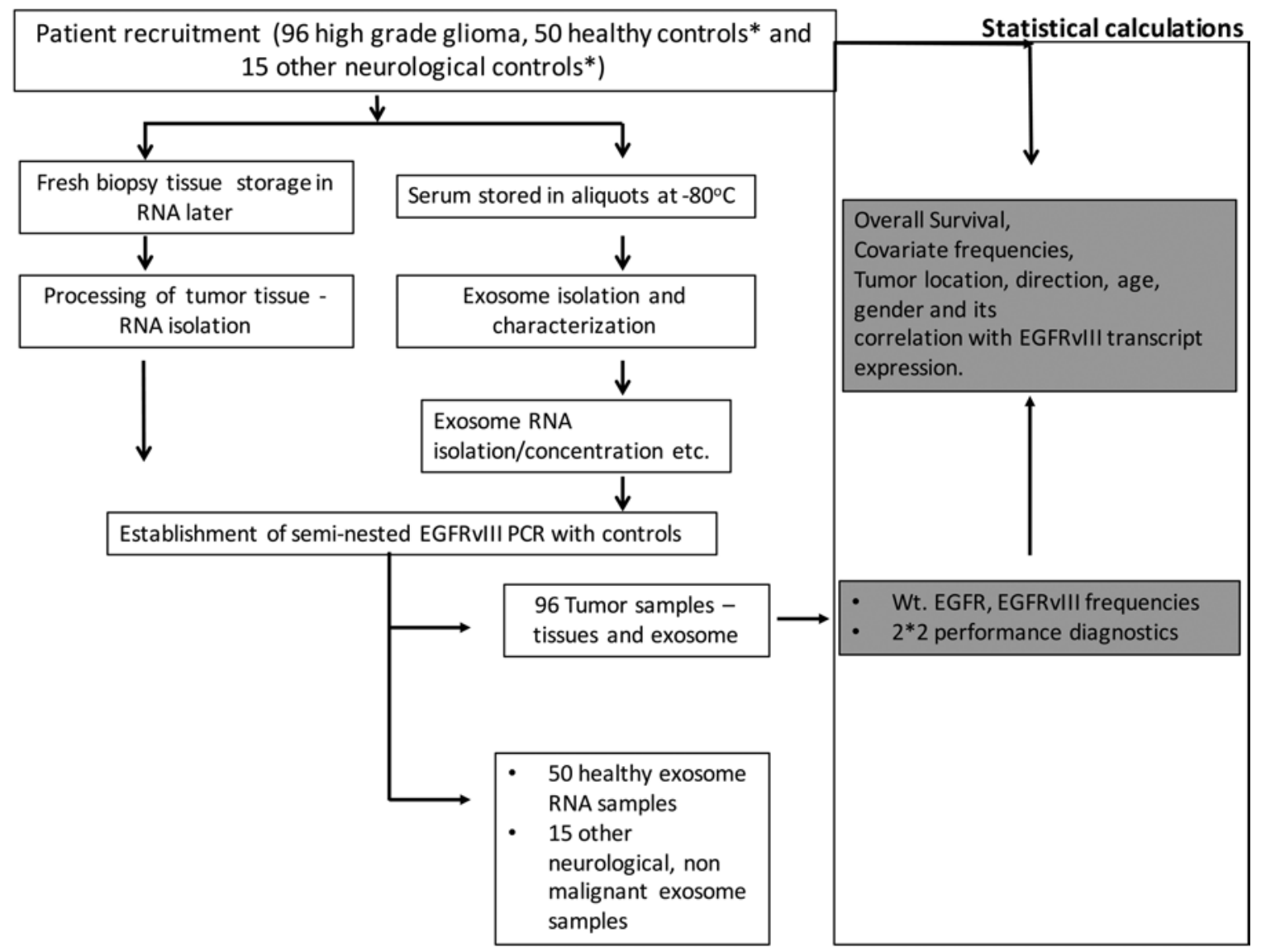

FIG. 1. Study design. Representative study design. Wt. = wild type. *Only serum samples.

\section{Transmission Electron Microscopy}

Transmission electron microscopy (TEM) was performed at the Center for Cellular and Molecular Biology, Hyderabad. Freshly isolated exosomes were dispensed on a 200-mesh copper grid (carbon coated), followed by glow discharge for 30 seconds using a Polaron carbon evaporator (Electron Microscopy Sciences). Five microliters of exosome samples was loaded on the glow-discharged grids and allowed to dry for 5 minutes. In addition, $5 \mu \mathrm{l}$ of uranyl acetate was loaded onto the grid after a 45 -second incubation. Excess uranyl acetate was wicked off using filter paper and air dried for 30 minutes. Samples were loaded on the TEM single tilt Holder (Gatan), loaded onto a JEM-2100Plus Transmission Electron Microscope (JEOL), imaged, and analyzed at $120 \mathrm{kV}$.

\section{Exosome Flow Cytometry}

Direct quantification of exosomes through traditional flow cytometers is a challenge because of their small size $(30-200 \mathrm{~nm})$. Therefore, in this study the protocol was slightly modified to indirectly quantify exosomes through quantification of the most common exosome surface marker, CD81. Briefly, $20 \mu \mathrm{g}$ of exosome protein was conjugated with $25 \mu \mathrm{l}$ of $4-\mu \mathrm{m}$ latex beads (ThermoFisher Scientific) in $100 \mu \mathrm{l}$ MES buffer and incubated overnight on a rotary shaker. The following day, the binding reaction was stopped by adding $100 \mathrm{mM}$ glycine for 30 minutes. Exosome-conjugated beads were washed in $0.1 \%$ bovine serum albumin wash buffer and conjugated with CD81 (BioLegend) and its corresponding isotope control (Mouse IgG1 к Isotype Control, BD Biosciences). Solutions were passed through a $0.2-\mu \mathrm{M}$ filter, and the fluorescence-activating cell sorter (FACS) instrument was cleaned twice to eliminate background noise. Exosome conjugated beads were acquired using BD FACSdiva (BD Biosciences) and analyzed with FlowJo (10.07, FlowJo LLC).

\section{RNA Handling and Storage}

Polymerase chain reaction (PCR) run conditions were designed to keep aerosol contamination to a minimum. RNase-free protocols were followed throughout all procedures, starting from PCR setup to PCR preparations. Dedicated, separate UV hoods were used for exosome and tissue RNA isolations. A dedicated laminar airflow cabinet was used for all PCR reaction preparation workups. All surfaces were thoroughly cleaned with $0.2 \%$ sodium dodecyl sulfate followed by $3 \% \mathrm{H}_{2} \mathrm{O}_{2}$. All pipettes and the hood floor were cleaned with RNase-zap (Sigma) to decrease contamination with external RNA. Furthermore, the laminar airflow cabinet is located in a Class 10,000 sterile facility. Double-gloving, regular changes, and clean laboratory coats were used. All reagents used in the PCR workup were RNA-free certified reagents. 


\section{RNA Isolation}

Tissue was homogenized, and then RNA was isolated using an RNAeasy Easy Lipid Tissue Kit (Qiagen) according to the manufacturer's instructions. Any DNA contamination was removed using the DNA-free RNA Kit (Zymo research). RNA was isolated from exosomes by using Total Exosome RNA and a protein isolation kit (ThermoFisher Scientific) as per the manufacturer's instructions. Ten micrograms of glycogen (ThermoFisher Scientific) was added during the RNA precipitation step after overnight incubation. RNA was eluted in 10-15 $\mu$ l of nucleic acidfree water supplied along with the aforementioned kit followed by quantification with a RiboGreen (ThermoFisher Scientific) assay according to the manufacturer's instructions. The exosome RNA quality, yield, and size were analyzed using capillary electrophoresis (2100 Bioanalyzer, Agilent Technologies). One microliter of exosome RNA was used with the Agilent RNA 6000 Pico Kit (catalog no. 5067-1513) according to the manufacturer's protocol. Electropherograms were analyzed using the Agilent 2100 Expert B.02.07 software.

\section{Reverse Transcription PCR}

One to $2 \mu \mathrm{g}$ of RNA isolated from either tissue or exosomes was reverse-transcribed using a high-capacity complementary DNA Reverse Transcription Kit (Life Technologies), according to the manufacturer's instructions. Around $0.5 \mu \mathrm{l}$ of cDNA was used as a template in the PCR reaction containing $0.2 \mu \mathrm{M}$ of each primer. Forward and reverse primer sequences for EGFR and EGFRvIII (primer set 1) were 5'CTTCGGGGAGCAGCGAT GCGAC3' and 5'ACCAATACCTATTCCGTTACAC3'.22 PCR was performed using a Master Cycler Pro S model (Eppendorf). From another set of primers (primer set 2), forward primer 5'ATGCGACCCTCCGGGACGGC3' was used, retaining the reverse primer mentioned above. PCR cycling conditions were the same for both primer set combinations: an initial denaturation step at $95^{\circ} \mathrm{C}$ for 5 minutes, followed by 37 cycles of denaturation at $95^{\circ} \mathrm{C}$ for 30 seconds, annealing at $58.5^{\circ} \mathrm{C}$ for 30 seconds, and extension at $68^{\circ} \mathrm{C}$ for 80 seconds. PCR products were analyzed on a $1.5 \%$ agarose gel. GAPDH (glyceraldehyde 3-phosphate dehydrogenase) was used as a loading control (forward 5'GAAGGTGAAGGTCGGAGTC3', reverse 5'TCAGAAGATGGGATTTTC3').

\section{Kaplan-Meier Survival Curves}

Tumors were graded by the pathology team according to WHO guidelines. ${ }^{20}$ OS (Grade III vs Grade IV) was calculated at the last follow-up or from the date of surgery to death. Death was the only defined event after surgery. Comparison of survival curves of patients with the EGFRvIII messenger RNA transcript in tissues and exosomes was done using the log-rank test (Mantel-Cox); $\mathrm{p}<0.05$ was considered statistically significant. The prognostic relevance with regard to OS of these parameters (e.g., grade, age, sex, EGFRvIII [tissue and exosome], and transcript presence or absence) was assessed. To compare the correlations between different groups, we performed a log-rank test (Mantel-Cox) of equality of survival dis-
TABLE 1. Clinicopathological characteristics of the 96 patients with high-grade gliomas

\begin{tabular}{lcc}
\hline \multicolumn{1}{c}{ Characteristics } & Grade III $(\mathrm{n}=23)$ & Grade IV $(\mathrm{n}=73)$ \\
\hline Frequency & $24.0 \%$ & $76.0 \%$ \\
\hline Median age in yrs* & $44(35-55)$ & $53(40-61)$ \\
\hline Sex & & \\
\hline Male & $19(82.6 \%)$ & $48(65.8 \%)$ \\
\hline Female & $4(17.4 \%)$ & $25(34.2 \%)$ \\
\hline
\end{tabular}

* Presented with the IQR.

tributions for the different tumor grades and the effect on OS of age, sex, and the presence or absence of EGFRvIII in tissues and exosomes.

\section{Statistical Analysis}

Statistical analysis was performed using SPSS (version 16.0, SPSS Inc.) and GraphPad (Prism). All continuous variables were assessed for normality using the ShapiroWilk's test. All values are expressed as mean \pm SD if they followed a gaussian distribution. All categorical variables are expressed as percentages. Comparisons of normally distributed continuous variables were performed using an independent sample t-test. Comparisons of categorical variables were performed using the chi-square test or Fisher's exact test based on the number of observations. Sensitivity, specificity, positive predictive values (PPVs), and negative predictive values (NPVs) with $95 \%$ confidence intervals were computed. Kaplan-Meier survival curves were plotted, and differences in survival between various groups were compared using the log-rank test. To measure the level of agreement, the kappa value was computed; $\mathrm{p}<0.05$ was considered statistically significant.

\section{Results}

\section{Study Population}

A total of 96 patients with high-grade glioma, 50 ageand sex-matched healthy controls, and 15 controls with neurological diseases (nonglioma) were recruited from Apollo Hospitals, Hyderabad, India. Table 1 summarizes the clinicopathological features of tumor patients used in the study. Healthy controls were sex and age matched to the tumor patients. All samples were anonymized for this study. All tissue specimens acquired at initial diagnosis and resection were classified morphologically and graded according to $\mathrm{WHO}$ criteria by the histopathologists. Investigators establishing the assay were blinded to the tumor grade and the patient's outcome. The patients ranged in age from 20 to 75 years old at the time of primary surgery (median age 53 years for patients with Grade IV tumors and 44 years for those with Grade III tumors); $24 \%$ of patients harbored a Grade III glioma, and 76\% harbored a Grade IV glioma.

\section{Characterization of Microvesicles Isolated From Serum From High-Grade Glioma Patients}

To detect EGFRvIII in serum exosomes of high-grade 


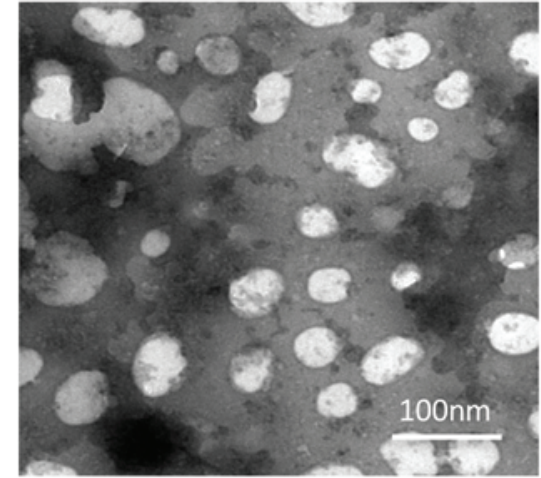

A

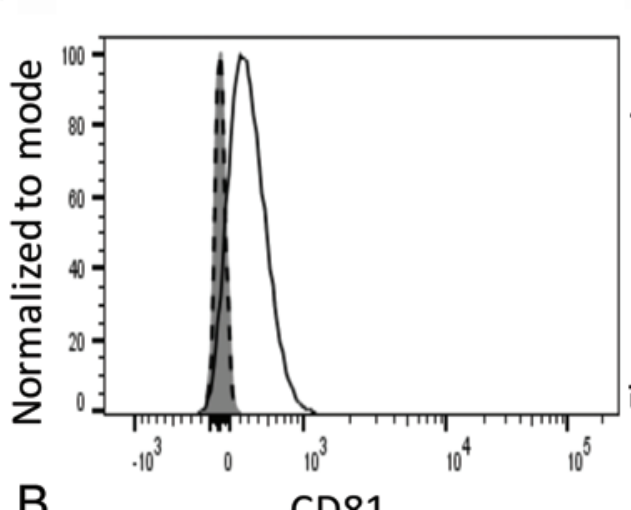

CD81

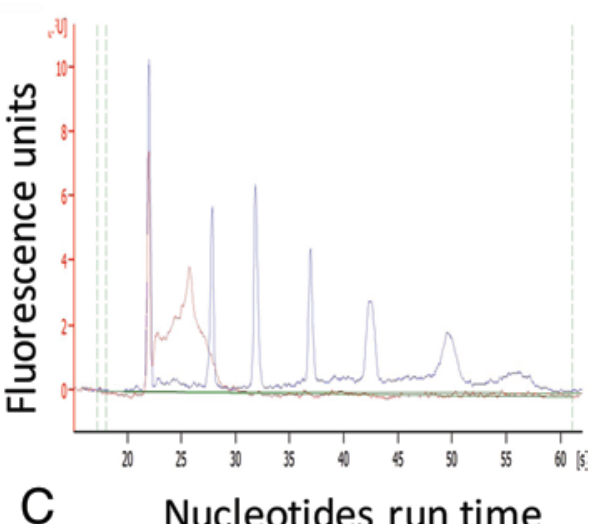

Nucleotides run time

FIG. 2. Characterization of exosomes/exosomal RNA from serum exosomes. A: Representative TEM image of an exosome obtained from a high-grade glioma patient and suspended in $1 \%$ glutaraldehyde $(n=6)$. B: Representative flow cytometry overlay of CD81 fluorescence (solid line) on exosomes overlaid with isotype control (dashed line) and solid bead fluorescence (solid peak) (n $=20$ ). C: Representative overlay of total exosomal RNA isolated from serum obtained in a high-grade glioma patient. An exosomal RNA electrophoretogram is overlaid with RNA standard for size estimation $(n=15)$. Figure is available in color online only.

glioma patients, we first isolated and characterized exosomes. Exosomes were isolated using commercial kits. The morphology of exosomes was confirmed by TEM (Fig. 2A). Double-membrane, round, intact structures in the range of 50-200 $\mathrm{nm}$ were observed. Furthermore, we performed flow cytometry to quantify the expression of the pan-exosome-specific marker CD81. Figure 2B clearly shows the expression of CD81 when compared with bead autofluorescence and isotype control staining. After morphological and biophysical/biochemical characterization of exosomes, we isolated RNA from these exosomes under RNase-free conditions using commercially available kits. Exosomal RNA was in the size range of 20-250 nucleotides (Fig. 2C). On the basis of these results, we confirmed the isolation and purity of exosome/exosomal RNA as per the parameters described in the published literature about the nature of exosomes and their RNA.

\section{Establishment of EGFRvIll and Wild-Type EGFR Semiquantitative Reverse Transcription PCR}

Given that exosomes are very small and the amount of isolated RNA was very low, we used semi-nested PCR (2-4 $\mu \mathrm{l}$ of the first-step PCR product is used as a template for the second-step PCR amplification) to amplify the EGFR variant.

$E G F R$ primers (spanning the $5^{\prime}$ untranslated region and the beginning of exon 1 and within exon 9) were used to amplify both the wild-type EGFR and EGFRvIII variants in a semiquantitative reverse transcription (RT)-PCR. With the use of primer set 1, these primers generated a 1044-bp PCR product for the wild-type EGFR, compared with a 243-bp PCR product for its splice variant EGFRvIII (a difference of $801 \mathrm{bp}$ ) (Fig. 3). For primer set 2, wild-type EGFR yielded a band at $1029 \mathrm{bp}$, while EGFRvIII yielded a band at 228 bp (Supplementary Figure 2). RNA from the U87 cell line was used as a positive control for wildtype EGFR expression. U87-MG and U87MG-EGFRvIII cell lines and breast cancer samples were used as positive controls in establishment of the PCR assay.
RNA from primary breast cancer patients was used as a positive control for EGFRvIII expression. The amplified products were further sequenced to confirm the specific expression of wild-type EGFR and EGFRvIII.

\section{Assessment of Wild-Type EGFR and EGFRvIll in Biopsy Tissue and Paired Exosomes}

Once we optimized exosome isolation and PCR amplification of wild-type EGFR and EGFRvIII, we proceeded to test the expression of EGFRvIII in exosomes isolated from patients with high-grade gliomas. Analyses were performed in 96 patient samples. Exosomal expression of the EGFRvIII transcript was found in 43 of $96(44.79 \%)$ tumor patients, whereas it was expressed in 38 of 96 (39.6\%) tumor tissue samples. Among the tumor samples, 72 of $96(75.0 \%)$ were positive for wild-type EGFR expression. In 27 of $96(28.12 \%)$ patients with tumors, the tumor tissue samples coexpressed wild-type EGFR and EGFRvIII. The wild-type EGFR transcript was not detected in any of our exosome fractions, which may be due to the nature of small RNA present in the exosomes. ${ }^{31}$ Sex, age, and side of the body where the tumor was located (right or left) did not significantly affect detection of exosome EGFRvIII expression. Frequency and tissue expression of wild-type EGFR and EGFRvIII for Grade III and IV gliomas are depicted in Table 2. Representation of EGFRvIII expression in both tissue and exosome fractions are represented in Fig. 3. GAPDH was used as an internal control. All experiments were repeated 3 times for confirmation.

\section{Diagnostic Evaluation Test}

The performance of the exosome EGFRvIII assay was statistically determined through clinical sensitivity, specificity, PPV, NPV, and EGFRvIII frequency. For diagnostic evaluation, we tested 96 tumor samples, 50 serum samples from healthy controls, and 15 serum samples from patients with nonmalignant neurological disease. Only the tumor and exosome EGFRvIII transcript data were obtained for statistical calculations. We used a clinical calculator 


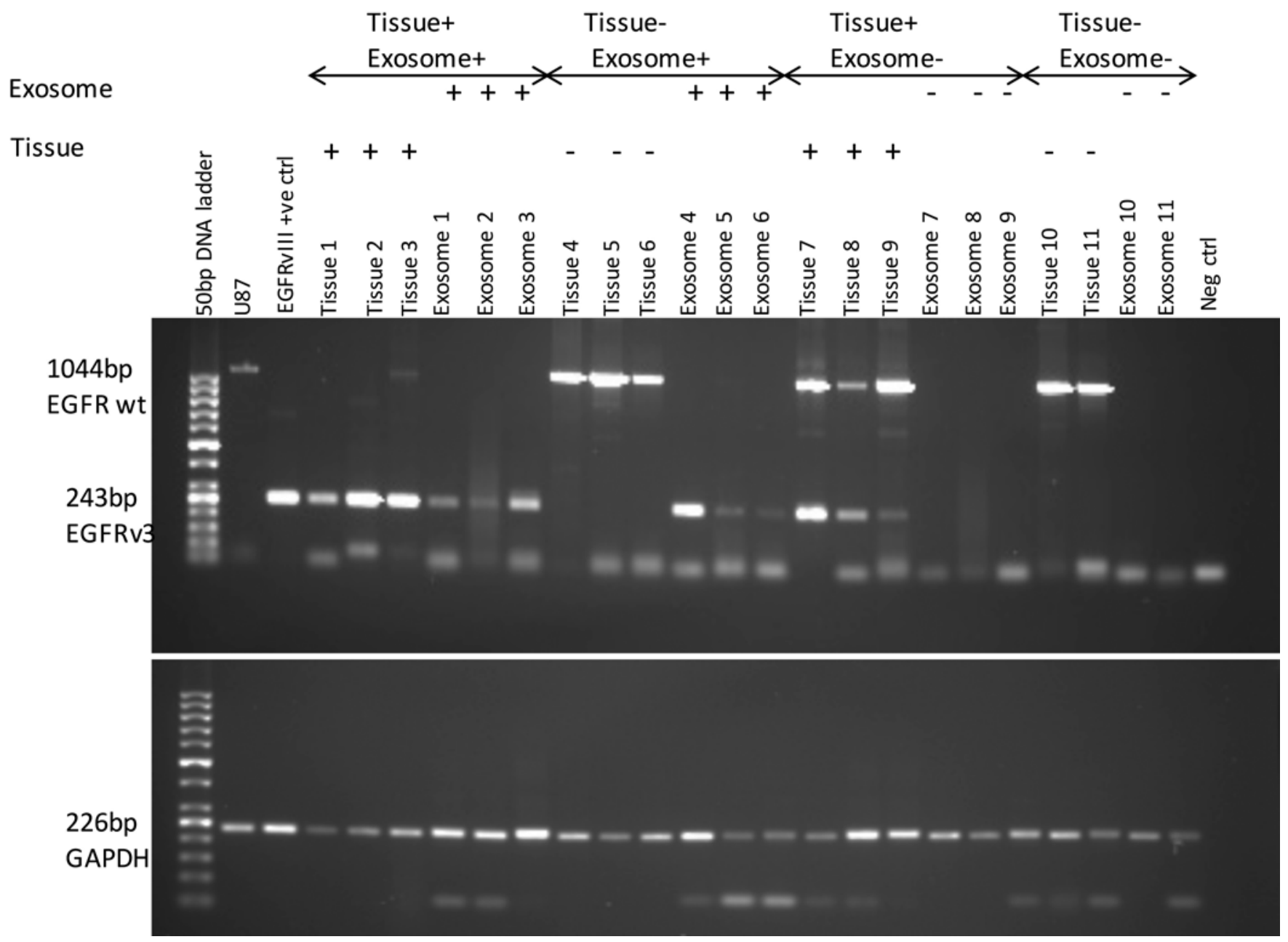

FIG. 3. EGFR/EGFRvIII expression in tissue and exosomal fractions. RT-PCR was used to detect EGFRvIII messenger RNA in the tumor samples, and semi-nested RT-PCR was used to detect EGFRvIll from its paired serum exosome RNA fraction. The wild-type EGFR PCR product appears as a band at $1044 \mathrm{bp}$ and its splice variant, EGFRvIII, at $243 \mathrm{bp}$. Lane 1 represents a 50-bp ladder, Lane 2 represents positive control for wild-type EGFR (U87 cell line), and Lane 3 represents positive control for EGFRVIII (high-grade glioma), confirmed by sequencing. The first group (Samples 1-3) represents positive EGFRvIII expression, both in tissues and exosomes. The second group (Samples 4-6) includes negative EGFRvIII expression in tissues and positive in exosomes. The third group (Samples 7-9) includes positive EGFRvIII expression in tissues and negative EGFRvIII expression in exosomes. The fourth group (Samples 10 and 11) includes double negative for EGFRvIll expression in tissues and exosomes. RT-PCR of GAPDH messenger RNA was included as a loading control (226 bp). Figure is representative of 96 glioma samples, repeated 3 times each. ctrl = control; $w t=$ wild type; + = present; $-=$ absent.

TABLE 2. Wild-type EGFR and EGFRvIll expression status in tumor patients

\begin{tabular}{lcc}
\hline \multirow{2}{*}{ Characteristic } & \multicolumn{2}{c}{ No. of Patients (\%) } \\
\cline { 2 - 3 } & Grade III $(\mathrm{n}=23)$ & Grade IV ( $=73)$ \\
\hline Exosome EGFRvIII & $9(39.1)$ & $34(46.6)$ \\
\hline Tissue biopsy EGFRvIII & $9(39.1)$ & $29(39.7)$ \\
\hline Tissue wild-type EGFR & $21(91.3)$ & $51(69.9)$ \\
\hline $\begin{array}{c}\text { Tissue biopsies coexpressing } \\
\text { wild-type EGFR \& EGFRvIII }\end{array}$ & $8(34.8)$ & $19(26.03)$ \\
\hline
\end{tabular}

based on a $2 \times 2$-table format for testing (https://www. medcalc.org/calc/diagnostic_test.php). All cases of detectable EGFRvIII messenger RNA in both exosome fraction and tissue biopsies were considered to be true positives; all cases of undetectable EGFRvIII in tissues and all cases of detectable EGFRvIII in exosomes were considered to be false positives; all cases of detectable EGFRvIII in tissues and all cases of undetectable EGFRvIII in exosomes were considered to be false negatives; all cases of undetectable EGFRvIII both in exosomes and tissue biopsy were considered to be true negatives (Table 3 ). The performance of the assay is shown in Table 4.

The sensitivity and specificity of the data ( 96 high-grade glioma samples) were $81.58 \%$ (95\% CI $65.67 \%-92.96 \%$ ) and $79.31 \%$ (95\% CI 66.65\%-88.83\%), respectively, while 
TABLE 3. The $2 \times 2$ diagnostic matrix for defining the 4 diagnostic groups

\begin{tabular}{ccc}
\hline Exosome & \multicolumn{2}{c}{ Tissue Biopsy Transcript } \\
\cline { 2 - 3 } Transcript & EGFRvIII $(+)$ & EGFRvIII $(-)$ \\
\hline EGFRvIII $(+)$ & True positive $(\mathrm{A})(\mathrm{n}=31)$ & False positive $(\mathrm{C})(\mathrm{n}=12)$ \\
\hline EGFRvIIII $(-)$ & False negative $(\mathrm{B})(\mathrm{n}=7)$ & True negative $(\mathrm{D})(\mathrm{n}=46)$ \\
\hline
\end{tabular}

+ = present; - = absent.

Letters A-D are used in Table 4.

the PPV and NPV were $72.09 \%$ (95\% CI 56.33\%-84.67\%) and $86.79 \%$ (95\% CI $76.87 \%-92.86 \%$ ), respectively. EGFRvIII prevalence (Table 4) in the data set was $39.58 \%$ (95\% CI $29.75 \%-50.08 \%, \mathrm{p}=0.0001)$.

Accuracy of the test was calculated using the following formula: (true positive + true negative) divided by (sum of all EGFRvIII positive + negative cases). Accuracy of EGFRvIII detection was $80 \% \pm 0.2 \%$. A statistically significant $(\mathrm{p}<0.0001)$ association between exosome and tissue biopsy EGFRvIII expression was observed on chisquare tests. In addition, there was a moderate agreement ( $\kappa=0.548)$ between exosome and tissue biopsy EGFRvIII expression $(\mathrm{p}<0.0001)$. The confidence intervals represented were "exact" Clopper-Pearson confidence intervals. In 12 patients EGFRvIII and GBM were detected in the serum exosome fraction and were undetectable in the tissue fraction. In addition, in 7 of the patients, EGFRvIII was undetectable in the serum exosome fraction even though it was detected in the tissue biopsy samples.

\section{Impact of EGFRvIll on OS}

No statistically significant difference in OS was observed between the Grade III and IV glioma patient groups (Fig. 4A). However, a clear trend toward worse prognosis was evident in the Grade IV glioma cohort. The mean survival durations for patients with Grade IV and Grade III tumors were 22.5 and 26.5 months, respectively ( $\mathrm{p}=$ 0.200, log-rank test).
In line with the previous findings, we observed that the patients with EGFRvIII present in the tumors had a worse prognosis. ${ }^{13}$ The mean OS durations for patients with and without exosome EGFRvIII transcript expression were 21.1 and 28.6 months, respectively ( $\mathrm{p}=0.005$; Fig. $4 \mathrm{~B})$. The mean OS durations for patients with and without tumor tissue EGFRvIII expression were 22.1 and 26.20 months, respectively ( $\mathrm{p}=0.032$ [Fig. $4 \mathrm{C}]$ ). The expression of EGFRvIII correlated with OS, suggesting that detection of EGFRvIII expression in either of these compartments would be sufficient for accurate diagnosis of GBMs (Fig. 4D). This effect was more pronounced when analysis was made through exosomes rather than tissues because of the increased detection rate. A multivariate survival model (Table 5) for OS was established that included pathology (Grades III and IV), age, sex, and EGFRvIII expression (tissue and exosome).

\section{Discussion}

It is well known that high-grade glioma cells use tumor exosomes as vehicles to transfer genetic information into blood and CSF. ${ }^{18}$ The internal cargo of the exosome consists of RNA, DNA, proteins, and other metabolites, which makes exosomes prime targets for interrogation in clinical diagnostics and therapeutics. ${ }^{29}$ EGFRvIII expression is unique to certain cancer types and serves as a good marker for their prognosis, diagnosis, and therapy monitoring and as a form of targeted therapy. ${ }^{19}$

Most of the protocols used for characterizing exosomes are research based and are not standardized for clinical adaptation. ${ }^{37} \mathrm{We}$ established a robust method incorporating 2 different primer sets to detect exosome EGFRvIII through serum exosomes (Fig. 3 and Supplementary Figure 1). We used a commercially available exosome-precipitating reagent to precipitate serum exosomes and ascertained their purity. Typically, the RNA we found in our exosome preparations was very small (25-200 nucleotides). Incidentally, we could only detect EGFRvIII and not the wild-type EGFR, which is in line

TABLE 4. Diagnostic performance of the exosome EGFRvIll assay

\begin{tabular}{llc}
\hline Diagnostic Parameter & \multicolumn{1}{c}{ Formula* $^{*}$} & Performance Characteristic $(95 \% \mathrm{Cl}) \dagger$ \\
\hline Sensitivity $\ddagger$ & A/A + B & $81.58 \%(65.67-92.26 \%)$ \\
\hline Specificity $\ddagger$ & D/C + D & $79.31 \%(66.65-88.83 \%)$ \\
\hline Positive likelihood ratio & Sensitivity/100 - specificity & $3.94(2.33-6.67)$ \\
\hline Negative likelihood ratio & $100-$ sensitivity/specificity & $0.23(0.12-0.46)$ \\
\hline EGFRvlll prevalence & $(A+B) /(A+B+C+D)$ & $39.58 \%(29.75-50.08 \%)$ \\
\hline PPV $\dagger$ & A/A + C & $72.09 \%(56.33-84.67 \%)$ \\
\hline NPV $\dagger$ & D/B + D & $86.79 \%(76.87-92.86 \%)$ \\
\hline
\end{tabular}

Sensitivity: probability that a test result will be positive when the EGFRvIII expression is present in both tissues and exosomes; specificity: probability that a test result will be negative when the expression is not detectable in both tissues and exosomes; positive likelihood ratio: how much more likely an individual with EGFRvIII expression is to have a positive test result than a person without it; negative likelihood ratio: how much more likely an individual without EGFRVIII is to have a negative test result, compared to a person with EGFRvIII expression; PPV: probability that EGFRvIII is detectable when present; NPV: probability that EGFRvIII is not detectable when absent.

* See Table 3 for definitions of Letters A-D.

$\dagger$ Confidence intervals are "exact" (Clopper-Pearson) confidence intervals.

$\ddagger$ Sensitivity, specificity, PPV, and NPV are expressed as percentages for ease of interpretation. 


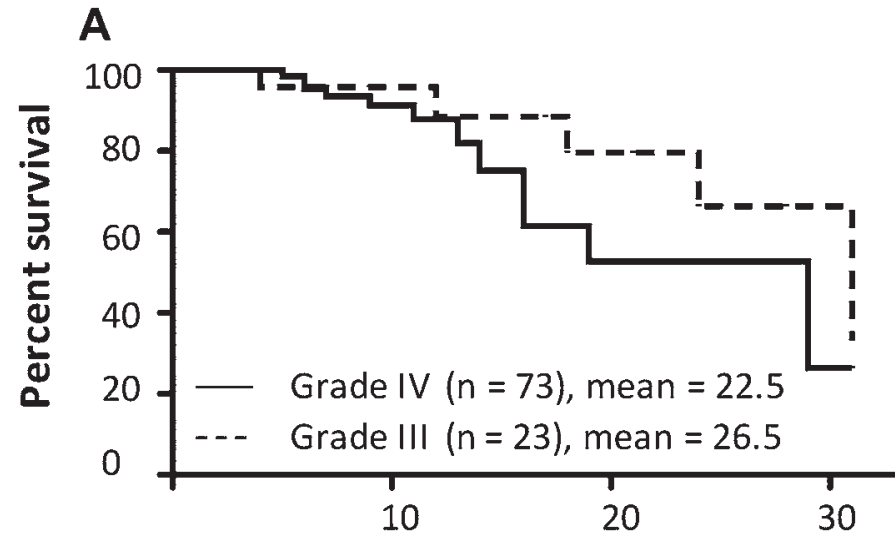

B

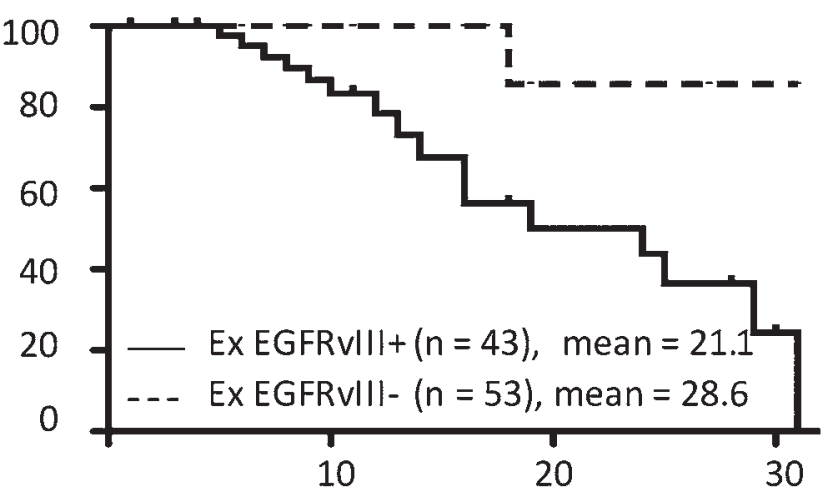

D

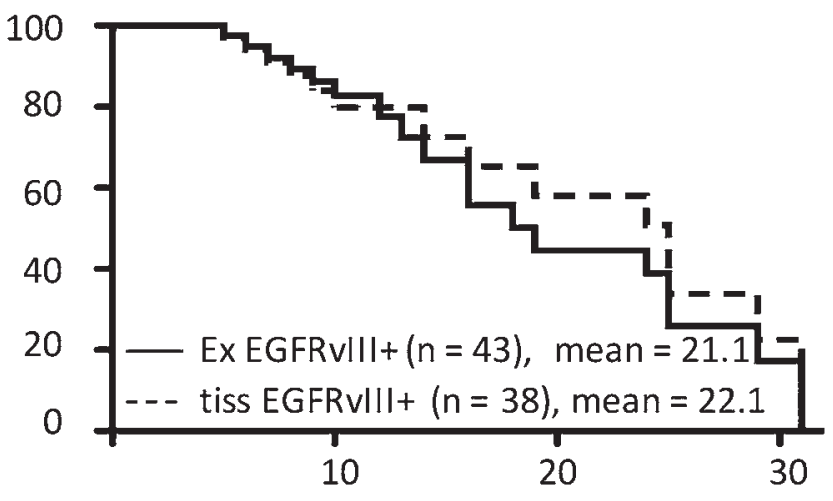

Months after surgery

\begin{abstract}
FIG. 4. Kaplan-Meier estimates of the OS of tumor patients in various EGFRvIII cohorts. A: OS of patients with Grade IV $(n=$ 73 ) and Grade III $(n=23)$ tumors. Mean OS times were 22.5 and 26.5 months, respectively $(p=0.200)$. B: OS of tumor patients with exosomal EGFRvIII transcript $(n=43)$ and patients devoid of exosomal EGFRvIII expression $(n=53)$. Mean OS times for the exosomal EGFRvIII-positive versus exosomal EGFRvIII-negative patient groups were 21.1 and 28.6 months, respectively $(p=0.005)$. C: OS of tumor patients with EGFRvIII transcript in tissues $(n=38)$ and patients devoid of tissue EGFRvIll $(n=58)$. Mean OS times for the tissue EGFRvIII-positive versus tissue EGFRvIII-negative patient groups were 22.1 and 26.2 months, respectively $(p=0.032)$. D: OS of tumor patients with EGFRvIII transcript in tissues $(n=38)$ and patients with exosomal EGFRvIII transcript $(n=43)$. Mean OS durations for the exosomal EGFRvIII and tissue EGFRvIll patient groups were 21.1 and 22.1 months, respectively $(p=0.2556)$. Ex $=$ exosomal; tiss $=$ tissue.
\end{abstract}

with previous findings that wild-type EGFR is undetectable in exosomes probably due to the larger size of the transcript. ${ }^{31}$ Accuracy of EGFRvIII detection through exosomes was $80 \%$ for tissue EGFRvIII expression with an overall sensitivity and specificity of $81.58 \%$ and $79.31 \%$, respectively.

In this study, the tumor tissue EGFRvIII expression frequency was $39.58 \%$. These results are in agreement with earlier studies that have reported EGFRvIII tissue expression in about $24 \%-67 \%$ of GBMs ${ }^{13}$ Additionally, the PPV and NPV were $72.09 \%$ and $86.79 \%$, respectively. PPV and NPV are considered good indicators of the accuracy of diagnostic procedures. The present study builds on previous findings ${ }^{11}$ with significant modifications in the PCR analytical methodology, associated with exosome RNA processing/handling for biomarker analysis. Glycogen addition during exosome RNA precipitation, exosomal RNA concentration, exosomal DNA contamination removal, and introduction of a semi-nested PCR to amplify exosome EGFRvIII expression during analysis were the major changes that were incorporated into the EGFRvIII detection protocol. In addition, extensive care was taken to decrease aerosol contamination by working in different UV hoods throughout the entire procedure and working with multiple serum aliquots. To overcome the problem of complementary DNA degradation in exosome preparations, we made multiple aliquots of complementary DNA to avoid repeated freeze-thaw procedures.

In total, we observed 12 cases of false positives, for which EGFRvIII was not detected in tissues but could be detected in the exosomes. Given that high-grade glial tumors exhibit considerable inter- and intratumoral heterogeneity, ${ }^{25}$ it is possible that the representative sample from which tissue RNA was isolated may not reflect the true molecular pathophysiology of the patient. Furthermore, some of the tumor tissue fractions from which we had extracted the RNA had considerable amounts of necrosis, which also might impact the detection ability of EGFRvIII in tissues. Thus, blood-based detection of EGFRvIII from the exosomal fraction could potentially mitigate the di- 
TABLE 5. Multivariate survival model for OS

\begin{tabular}{|c|c|c|c|c|c|}
\hline Parameter & $\begin{array}{c}\text { No. of } \\
\text { Patients }\end{array}$ & $\begin{array}{l}\text { No. of } \\
\text { Events }\end{array}$ & $\begin{array}{c}\text { No. Censored } \\
(\%)\end{array}$ & $\begin{array}{c}\text { Mean OS } \\
(\mathrm{mos})^{*}\end{array}$ & $\begin{array}{c}\mathrm{p} \\
\text { Valuet }\end{array}$ \\
\hline All patients & 96 & & & & \\
\hline Grade & & & & & 0.200 \\
\hline III & 23 & 5 & $18(78.3)$ & 26.538 & \\
\hline IV & 73 & 11 & $62(84.9)$ & 22.532 & \\
\hline $\begin{array}{l}\text { Tissue } \\
\text { EGFRvIII }\end{array}$ & & & & & 0.032 \\
\hline Present & 38 & 12 & $26(68.4)$ & 22.09 & \\
\hline Absent & 58 & 4 & 54 (93.1) & 26.20 & \\
\hline $\begin{array}{l}\text { Exosome } \\
\text { EGFRvIII }\end{array}$ & & & & & 0.005 \\
\hline Present & 43 & 14 & $29(67.4)$ & 21.13 & \\
\hline Absent & 53 & 2 & $51(96.2)$ & 28.57 & \\
\hline Age (yrs) & & & & & 0.317 \\
\hline$<50$ & 48 & 7 & $41(85.4)$ & 25.5 & \\
\hline$\geq 50$ & 48 & 9 & 39 (81.2) & 21.1 & \\
\hline Sex & & & & & 0.883 \\
\hline Male & 67 & 10 & 57 (85.1) & 23.53 & \\
\hline Female & 29 & 6 & $23(79.3)$ & 24.94 & \\
\hline
\end{tabular}

agnostic hindrances associated with tumor heterogeneity. Seven cases of false negatives were observed, wherein EGFRvIII was not detected in the exosomes, but was detected in tissue biopsies. Although exosomes were discovered a while ago, the recognition of their clinical potential as biomarker shuttles is very recent. ${ }^{16}$ Exosomes, being very small, contain a minute amount of RNA and therefore pose significant challenges in detection of their specific markers. Furthermore, the limited availability of standardized protocols for exosome isolation and subsequent RNA isolation is also a matter of concern. ${ }^{37}$ It has been recently reported that diurnal variations influence exosome secretion into the peripheral circulation, and factors such as diet, exercise, and circadian variations do have an effect on their detectability?

It is known that the sensitivity of the detection of exosomes or any circulatory biomarker may depend on various tumor intrinsic and extrinsic factors, such as tumor size, location, serum volume, RNA extraction method, and complementary DNA conversion procedures. However, in our study, age, sex, and tumor location did not show any impact on exosome EGFRvIII detection (Supplementary Figure 3).

The expression of EGFRvIII transcript in exosomes mirrored the OS pattern. Moreover, though not significant, the OS pattern correlated with the aggressive nature of Grade IV tumors over Grade III tumors (Fig. 4A). OS curves indicated worse prognosis in patients who harbored EGFRvIII (either in exosomes or tissue fractions) than in those patients who did not. Furthermore, OS curves of patients with EGFRvIII in their tissue biopsies and those with exosomal EGFRvIII mirrored each other, suggesting that either tissue- or serum-derived exosomes can be reliably employed for diagnosis (Fig 4D). Age and sex did not have significant effects on OS in our cohort, which is consistent with previous findings that these factors are not predictors of OS in patients harboring high-grade tumors. ${ }^{14}$

This study could provide a platform for blood-based diagnosis of high-grade glial tumors by detection of EGFRvIII alone or as a part of a host of biomarkers. This protocol can also be used in patient stratification for EGFRvIII-targeted therapies like mAB806, rindopepimut, and other small peptides targeting EGFRvIII. It is known that EGFRvIII mutation is associated with the "classical" GBM subtype ${ }^{35}$ and is an active target for various immuno-oncology drugs and immunotherapeutic agents. ${ }^{11}$ As the downstream signaling components of EGFRvIII are different from the wild-type EGFR, EGFRvIII detection may also be used for predicting the therapeutic response to targeted therapies to EGFR. ${ }^{10}$

Taken together, our results indicate that exosome-based biomarker diagnosis is a promising tool to circumvent tissue biopsy in patients who carry a very high surgical risk. Although these results are promising and substantiate the utility of exosomes as a source of biomarkers for detection of brain tumors, we recognize that this study does have some limitations. The sample size requires verification in a larger cohort of patients, with longitudinal controls to confirm the clinical utility of this assay technology. Furthermore, to increase the sensitivity of the assay, purification of glioma-specific exosomes may be a viable option, as demonstrated in Alzheimer's disease biomarkers through plasma neuronal exosomes.' Our analysis procedure needs to be extended to incorporate digital PCR methodology, which would further increase the sensitivity of detection, as has been shown in the case of detection of IDHI through CSF exosomes. ${ }^{4}$ In addition, a molecular signature/panel of biomarkers would add more value in establishing liquid biopsy than a single biomarker (EGFRvIII) and aid in the identification of TCGA subgroups. ${ }^{30}$ Thus, detection of other circulatory biomarkers (e.g., cell-free DNA, circulatory tumor cells, and microRNA) can complement medical imaging and add more specificity to the overall detection capability.

Our inclusion of a control group of patients with other CNS complications (e.g., abscesses, stroke, meningitis, and brain injury) that may mimic GBM on neuroimaging strongly substantiates the relevance of our study. Furthermore, we tested 50 healthy volunteers who did not have EGFRvIII expression (Supplementary Figure 2), which is in line with previous studies. ${ }^{10}$

\section{Conclusions}

Using exosome-based liquid biopsy to measure EGFRvIII expression for diagnosis of high-grade gliomas is highly promising and may help differentiate highgrade gliomas from infective and demyelinating diseases that may have a similar radiological picture. Exosomal biomarker evaluation may provide an effective means of deciphering a tumor's genetic profile and progression. Establishment of the clinical utility of this technology and 
validation of its diagnostic accuracy may lead to a redefinition of the standard of care in glioma patients.

\section{Acknowledgments}

This work was supported by a research grant from Apollo Hospitals Educational and Research foundation (AHERF). We acknowledge the AHERF staff for their patient outreach and logistic support. We thank the AHERF CEO, Mr. Seemant Jauhari, for his active organizational support. We thank Mr. Hari Krishna for his technical expertise in conducting electron microscope studies. We thank Dr. Swarna Latha and the Department of Pathology for their clinical investigations. We thank Apollo Hospitals President Dr. K. Hari Prasad for allowing us to access Apollo Hospitals patients to participate in the study.

\section{References}

1. Abner EL, Jicha GA, Shaw LM, Trojanowski JQ, Goetzl EJ: Plasma neuronal exosomal levels of Alzheimer's disease biomarkers in normal aging. Ann Clin Transl Neurol 3:399-403, 2016

2. Altman DG, McShane LM, Sauerbrei W, Taube SE: Reporting recommendations for tumor marker prognostic studies (REMARK): explanation and elaboration. PLoS Med 9:e1001216, 2012

3. An T, Qin S, Xu Y, Tang Y, Huang Y, Situ B, et al: Exosomes serve as tumour markers for personalized diagnostics owing to their important role in cancer metastasis. J Extracell Vesicles 4:27522, 2015

4. Chen WW, Balaj L, Liau LM, Samuels ML, Kotsopoulos SK, Maguire CA, et al: BEAMing and droplet digital PCR analysis of mutant IDH1 mRNA in glioma patient serum and cerebrospinal fluid extracellular vesicles. Mol Ther Nucleic Acids 2:e109, 2013

5. Choi BD, Archer GE, Mitchell DA, Heimberger AB, McLendon RE, Bigner DD, et al: EGFRvIII-targeted vaccination therapy of malignant glioma. Brain Pathol 19:713-723, 2009

6. Colombo M, Raposo G, Théry C: Biogenesis, secretion, and intercellular interactions of exosomes and other extracellular vesicles. Annu Rev Cell Dev Biol 30:255-289, 2014

7. Danielson KM, Estanislau J, Tigges J, Toxavidis V, Camacho $\mathrm{V}$, Felton EJ, et al: Diurnal variations of circulating extracellular vesicles measured by nano flow cytometry. PLoS One 11:e0144678, 2016

8. Del Vecchio CA, Jensen KC, Nitta RT, Shain AH, Giacomini $\mathrm{CP}$, Wong AJ: Epidermal growth factor receptor variant III contributes to cancer stem cell phenotypes in invasive breast carcinoma. Cancer Res 72:2657-2671, 2012

9. Erpolat OP, Akmansu M, Goksel F, Bora H, Yaman E, Büyükberber S: Outcome of newly diagnosed glioblastoma patients treated by radiotherapy plus concomitant and adjuvant temozolomide: a long-term analysis. Tumori 95:191-197, 2009

10. Gan HK, Kaye AH, Luwor RB: The EGFRvIII variant in glioblastoma multiforme. J Clin Neurosci 16:748-754, 2009

11. Greenall SA, Johns TG: EGFRvIII: the promiscuous mutation. Cell Death Dis 2:16049, 2016

12. Hatanpaa KJ, Burma S, Zhao D, Habib AA: Epidermal growth factor receptor in glioma: signal transduction, neuropathology, imaging, and radioresistance. Neoplasia 12:675-684, 2010

13. Heimberger AB, Hlatky R, Suki D, Yang D, Weinberg J, Gilbert M, et al: Prognostic effect of epidermal growth factor receptor and EGFRvIII in glioblastoma multiforme patients. Clin Cancer Res 11:1462-1466, 2005

14. Iliadis G, Kotoula V, Chatzisotiriou A, Televantou D,
Eleftheraki AG, Lambaki S, et al: Volumetric and MGMT parameters in glioblastoma patients: survival analysis. BMC Cancer 12:3, 2012

15. Karsy M, Gelbman M, Shah P, Balumbu O, Moy F, Arslan E: Established and emerging variants of glioblastoma multiforme: review of morphological and molecular features. Folia Neuropathol 50:301-321, 2012

16. Kawikova I, Askenase PW: Diagnostic and therapeutic potentials of exosomes in CNS diseases. Brain Res 1617:6371,2015

17. Keller S, Ridinger J, Rupp AK, Janssen JW, Altevogt P: Body fluid derived exosomes as a novel template for clinical diagnostics. J Transl Med 9:86, 2011

18. Lee Y, El Andaloussi S, Wood MJ: Exosomes and microvesicles: extracellular vesicles for genetic information transfer and gene therapy. Hum Mol Genet 21 (R1):R125R134, 2012

19. Lo HW: EGFR-targeted therapy in malignant glioma: novel aspects and mechanisms of drug resistance. Curr Mol Pharmacol 3:37-52, 2010

20. Louis DN, Perry A, Reifenberger G, von Deimling A, Figarella-Branger D, Cavenee WK, et al: The 2016 World Health Organization Classification of Tumors of the Central Nervous System: a summary. Acta Neuropathol 131:803820,2016

21. Lyons MK, Vora SA: Brain tumors: current issues in diagnosis and management. Semin Neurol 27:312-324, 2007

22. Mellinghoff IK, Wang MY, Vivanco I, Haas-Kogan DA, Zhu S, Dia EQ, et al: Molecular determinants of the response of glioblastomas to EGFR kinase inhibitors. N Engl J Med 353:2012-2024, 2005

23. Mischel PS, Cloughesy T: Using molecular information to guide brain tumor therapy. Nat Clin Pract Neurol 2:232233, 2006

24. Nagane M, Levitzki A, Gazit A, Cavenee WK, Huang HJ: Drug resistance of human glioblastoma cells conferred by a tumor-specific mutant epidermal growth factor receptor through modulation of Bcl-XL and caspase-3-like proteases. Proc Natl Acad Sci U S A 95:5724-5729, 1998

25. Nicholas MK, Lukas RV, Chmura S, Yamini B, Lesniak M, Pytel P: Molecular heterogeneity in glioblastoma: therapeutic opportunities and challenges. Semin Oncol 38:243-253, 2011

26. Nilsson RJ, Balaj L, Hulleman E, van Rijn S, Pegtel DM, Walraven M, et al: Blood platelets contain tumor-derived RNA biomarkers. Blood 118:3680-3683, 2011

27. Ohgaki H, Kleihues P: Genetic profile of astrocytic and oligodendroglial gliomas. Brain Tumor Pathol 28:177-183, 2011

28. Parker NR, Khong P, Parkinson JF, Howell VM, Wheeler HR: Molecular heterogeneity in glioblastoma: potential clinical implications. Front Oncol 5:55, 2015

29. Raposo G, Stoorvogel W: Extracellular vesicles: exosomes, microvesicles, and friends. J Cell Biol 200:373-383, 2013

30. Redzic JS, Ung TH, Graner MW: Glioblastoma extracellular vesicles: reservoirs of potential biomarkers. Pharm Genomics Pers Med 7:65-77, 2014

31. Shao H, Chung J, Lee K, Balaj L, Min C, Carter BS, et al: Chip-based analysis of exosomal mRNA mediating drug resistance in glioblastoma. Nat Commun 6:6999, 2015

32. Skog J, Würdinger T, van Rijn S, Meijer DH, Gainche L, Sena-Esteves M, et al: Glioblastoma microvesicles transport RNA and proteins that promote tumour growth and provide diagnostic biomarkers. Nat Cell Biol 10:1470-1476, 2008

33. Sturm D, Bender S, Jones DT, Lichter P, Grill J, Becher O, et al: Paediatric and adult glioblastoma: multiform (epi)genomic culprits emerge. Nat Rev Cancer 14:92-107, 2014

34. Valadi H, Ekström K, Bossios A, Sjöstrand M, Lee JJ, Lötvall JO: Exosome-mediated transfer of mRNAs and microRNAs 
is a novel mechanism of genetic exchange between cells. Nat Cell Biol 9:654-659, 2007

35. Verhaak RG, Hoadley KA, Purdom E, Wang V, Qi Y, Wilkerson MD, et al: Integrated genomic analysis identifies clinically relevant subtypes of glioblastoma characterized by abnormalities in PDGFRA, IDH1, EGFR, and NF1. Cancer Cell 17:98-110, 2010

36. Verma N, Cowperthwaite MC, Burnett MG, Markey MK: Differentiating tumor recurrence from treatment necrosis: a review of neuro-oncologic imaging strategies. Neuro Oncol 15:515-534, 2013

37. Witwer KW, Buzás EI, Bemis LT, Bora A, Lässer C, Lötvall $\mathrm{J}$, et al: Standardization of sample collection, isolation and analysis methods in extracellular vesicle research. J Extracell Vesicles 2:20360, 2013

\section{Disclosures}

The authors report no conflict of interest concerning the materials or methods used in this study or the findings specified in this paper.

\section{Author Contributions}

Conception and design: Ray, Manda, Ranjan. Acquisition of data: Kataria, Tatireddy. Analysis and interpretation of data: Ray, Manda, Kataria, Ratnam, Lath. Drafting the article: Ray, Manda. Critically revising the article: Ray, Manda. Approved the final version of the manuscript on behalf of all authors: Ray. Statistical analysis: Ramakrishnan. Administrative/technical/material support: Kataria, Tatireddy, Ratnam, Lath. Study supervision: Manda.

\section{Supplemental Information \\ Online-Only Content}

Supplemental material is available with the online version of the article.

Supplementary Figs. 1-3. https://thejns.org/doi/suppl/10.3171/ 2016.11.JNS161187.

\section{Correspondence}

Amitava Ray, Department of Neurosurgery, Rm. 953, International Block, Apollo Hospitals, Jubilee Hills, Hyderabad, Telangana, India. email: amitray0407@gmail.com. 